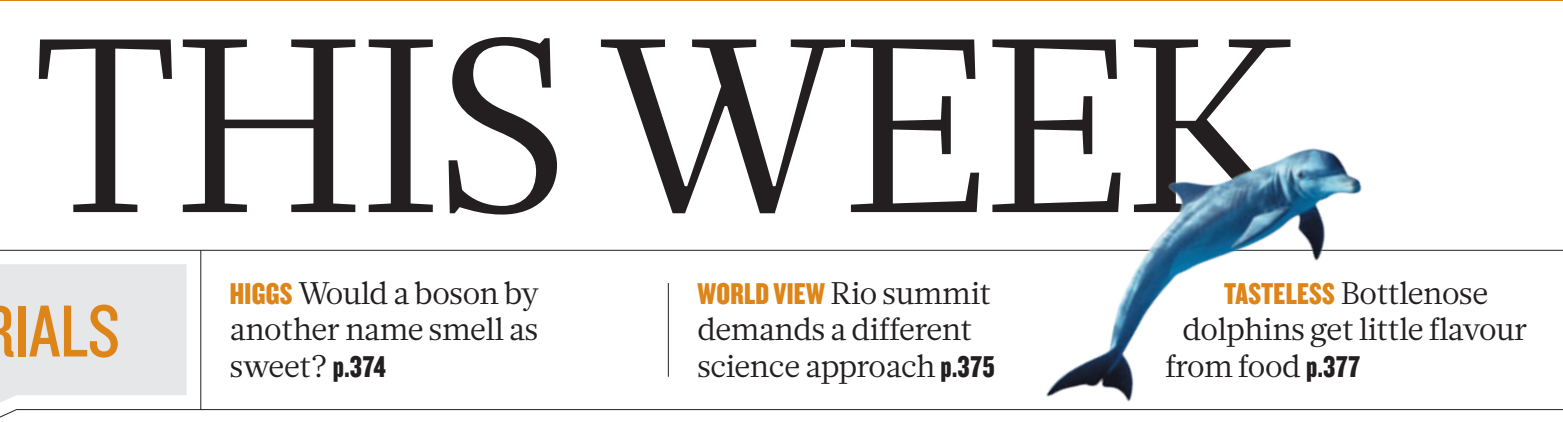

EDITORIALS

another name smell as sweet? p.374 mit cience approach $\mathbf{p . 3 7}$ dolphins get little flavour from food $\mathbf{p . 3 7 7}$

\title{
Incidental benefits
}

\section{Scientists who screen the genes of volunteers for research should tell participants if they find information relevant to their health.}

A 11 research studies on humans can uncover facts relevant to a volunteer's health - at initial screening, during the study itself, or even after the study finishes, when other researchers review the data or conduct their own analyses.

For the most part, researchers have opted not to reveal these potentially important 'incidental findings' to participants. This has been to protect the research process, and to prevent coercing people into studies by unwittingly eliciting the 'therapeutic misconception' - the incorrect assumption on the individual's part that participating in a study will help their own health.

But the emergence of high-throughput genomics, with its ability to catalogue vast amounts of information that may have a bearing on a person's health, has prompted a rethink of this convention.

A working group funded by the US National Institutes of Health (NIH) in Bethesda, Maryland, has recommended that biobanks and archives that house large genetic data sets introduce policies to encourage the return of incidental findings to research subjects (see page 387 ).

The impact of these recommendations should not be underestimated. As genetics invades every branch of medicine, no field is likely to be exempt from ethical standards introduced to cover genetic data. The recommendations are likely to have their most immediate influence in discussions on the topic now under way at the US Department of Health and the NIH.

There is a precedent for returning information to the subjects of a study. In imaging studies, for instance, a radiologist often reviews patients' scans for incidental findings before they are analysed by researchers. Support is growing for the idea that genetics researchers should similarly review a selected set of genes with known impacts on health before undertaking their own research.

Implementing this will not be easy. Defining appropriate sets of genes is problematic, and any list will need to be constantly updated. And to return the information to an individual in a way that avoids unnecessary anxiety and medical expense is a huge issue. Opponents of the idea point out that it contravenes standard practice in medicine itself, because doctors do not routinely sift through patients' records for old test results that may carry new significance in the light of more recent research.

Perhaps the most visible example of the need for this debate comes not from science, but from commerce. Companies routinely mine vast quantities of consumer information to influence marketing decisions. Governments have not been able to keep pace with standard business practices, and most consumers are unaware of the breadth and depth of information that companies gather on them from Internet searches, social networks and supermarket purchases.

In testimony before the US Presidential Commission on Bioethics in February, John Wilbanks, who runs the project Consent to Research, noted that this pattern is likely to repeat itself in the era of electronic medical records and genomics. "My sad realization is that whether it's your genome or your health information, anyone who really wants to

screw you will probably be able to get a copy of the data they need to do so, and the people who are least likely to get a copy are the people who can do something amazing with it, like researchers," he said. Companies are lining up to market products to consumers on the basis

"No field is likely to be exempt from ethical standards introduced to cover genetic data." of their genomes. Law-enforcement agencies already use DNA left at crime scenes to find suspects and their relatives, and are funding programmes to create physical profiles of suspects on the basis of their DNA. In other words, people now have incomplete protection for their own DNA, and this lack of privacy is likely to increase in the future.

In this free market, how sure can researchers be that they are truly doing no harm to their study participants when they take a cheek swab? People thinking of entering a study will assess the risks of how their volunteered genetic information might be used, and this might make them more reluctant to participate. Researchers could help to counter this by offering them medically relevant information back in exchange.

\section{Flight risk}

\section{As the campaign against animal research intensifies, so must the response.}

$\mathrm{P}$ icture a crowd of scientists waving placards plastered with photographs of stroke victims and sufferers of Parkinson's disease. They are demonstrating outside the corporate headquarters of British Airways, Lufthansa and Delta, demanding that the airlines stop impeding the biomedical research that could deliver big advances against these and other diseases.

Seem far-fetched? Maybe. But if scientists want continued access to animals as research models, they will have to appear on the front line with every bit as much visibility, determination, organization and persistence as animal-rights activists now muster.

In a renewed campaign targeting transportation companies, protestors have found a public pressure point so effective that only a few major airlines still agree to transport non-human primates bound for research labs (see page 381). Nor is the focus confined to primate transportation: earlier this year, the last ferry company that was willing to carry research rodents into the United Kingdom stopped doing so. Such blocks, scientists warn, could shift much animal work to countries where regulations are more lax.

But there is a silent majority for whom the activists do not speak. 
This includes most scientists, many ordinary citizens and millions of patients and their families touched by disease and injury. This constituency must mobilize in the defence of human health if the gains enabled by future primate research - and, ultimately, all animal research - are not to be thrown away.

Scientists and their allies must, of course, continue to be open about the price animals pay in research. They must openly acknowledge, immediately correct and do everything they can to prevent lapses in the care of the animals in their charge. At the same time, scientists must make every effort to use lower animal, and non-animal, models where possible, as regulations already require. And alongside all of that, they must emphasize the tangible and compelling improvements to human life that animal research has made possible.

Consider stroke, which affects some 795,000 people in the United States alone each year - 1 person every 40 seconds - at a cost in excess of US $\$ 40$ billion. More than 1,000 experimental treatments aimed at protecting brains cells in acute stroke have been developed in cells and rodents; none has been effective in humans. So a possible advance in a paper published last month is significant (D. J. Cook et al. Nature 483, 213-217; 2012). Using macaques - animals whose neuroanatomy, genetics and behaviour are far closer to humans than are those of rodents - the study showed that a drug called a PSD-95 inhibitor reduced the volume of brain tissue killed by the stroke and significantly preserved neurological function. It has now moved into human trials, where early results are promising.

Many other advances have been made possible by primate research. Trials of brain-machine interfaces that allow quadriplegics to control robotic limbs with their minds, for example, and of gene therapy for haemophilia B. And deep-brain stimulation to alleviate the symptoms of advanced Parkinson's disease. Primates have also helped the development of antiviral therapies to prevent mother-to-child transmission of HIV; of Rituxan (rituximab), a key drug against non-Hodgkin's lymphoma; and of Benlysta (belimumab), the first new lupus treatment in 50 years. Primates are making possible strides towards a vaccine against Ebola, and they have affirmed that the diabetes drug pioglita-
"Silence from the research community will mean lost access to research animals." zone can slow early Parkinson's disease - a therapy now being taken into human trials.

Future advances of this kind cannot be allowed to stall. The scientific community and its allies in patient and research advocacy groups should mount a vigorous, coordinated campaign to lobby major airlines. They should vocally support companies such as Air France that continue to fly research primates. They should confront others with the human cost of their alignment with animal activists. And to those airlines that are wavering, they should make a pointed, persuasive case.

An apt target in the last category is United Airlines, which merged with Continental in 2010. Continental was the last major US airline carrying non-human primates for research. The now-merged airline, still under the name of United, says it is "in the process of integrating" the former United and Continental policies on transporting research primates, for publication this autumn. Only a powerful and visible public campaign urging United to transport research primates will give the firm the political cover it needs to take such a position.

Urgent and dramatic action is necessary. It is increasingly clear that silence and passivity from the research community will lead to only one result: lost access to research animals in the countries best equipped for their responsible, humane and justified use.

\section{Mass appeal}

\section{As physicists close in on the Higgs boson, they should resist calls to change its name.}

\section{$\mathrm{T}$} The naming of the Higgs boson as such is clearly a simplification - physicists besides Peter Higgs contributed to the theory that predicts it. But it is far from the most extreme example.

The relationship between the velocity of recession of galaxies and their distance from Earth that we call Hubble's law was first formulated by Belgian cosmologist Georges Lemaitre. The quantity known as Avogadro's number was first calculated by Austrian chemist Johann Josef Loschmidt. Higgs, a physicist at the University of Edinburgh, $\mathrm{UK}$, at least has the strongest claim to credit for the boson. And for the arcane world of particle physics, simplification is often a good thing.

In 1964, Higgs was the first to postulate the existence of a massive particle arising from the mechanism of electroweak symmetry breaking, in which a unification of electromagnetism and the weak nuclear force fails in such a way as to give some force-carrying particles masses while others remain without. Yet moves are afoot to rename the Higgs. Earlier this month, some speakers at the Moriond particle-physics conference in La Thuille, Italy, chose to describe progress on the search for the BEH scalar boson (after the physicists Robert Brout, François Englert and Higgs) or the SM scalar boson (where SM stands for standard model).

It is not hard to guess why. Experiments at the Large Hadron Collider at CERN, Europe's premier particle-physics laboratory near Geneva in Switzerland, have reported tentative signals of the Higgs. If these are real, data collected in 2012 should see CERN claim a discovery. A Nobel prize is in the offing, and one that should arguably go not just to the experimentalists, but also to the theorists whose efforts inspired the successful search. But who - besides Higgs - should be included?

The name Higgs boson was supposedly coined by Korean-born physicist Ben Lee, some time between 1966 and 1972. According to journalist Ian Sample, Lee learned about the mass-giving mechanism from Higgs, and later coined the term as a shorthand. Yet several others played an important part in developing the theory that gives rise to the Higgs.

Belgian physicists Brout and Englert were the first to publish on the subject in 1964, building on ideas from condensed-matter physics developed by physics Nobel laureate Phil Anderson and others. Higgs published the same year; his paper contains the first mention of the boson. Tom Kibble, Gerald Guralnik and Carl Hagen followed with a third account that is generally considered more complete. One interpretation of this history is reflected in the American Physical Society's joint award, in 2010, of the J. J. Sakurai Prize for Theoretical Particle Physics to Brout, Englert, Higgs, Guralnik, Hagen and Kibble.

This provides plenty for physicists to argue about. In 2010, a row over credit erupted when Brout, Englert and Higgs were acknowledged in an advertisement for a conference on the particle but Guralnik, Hagen and Kibble were not (see Nature http://doi.org/ctz988; 2010). Meanwhile, 2011 saw a dispute over editing of the Wikipedia article 'Higgs boson' between one editor who supported Guralnik's view that his paper with Hagen and Kibble proposed a boson and another who was pro-Higgs.

Particle physicists should not rename the Higgs. And the reason would be obvious to anyone in business: branding. There are already relatively few concepts in their subject that have achieved widespread recognition without crossing one of them out. In business, it would be considered destructive to take a well-known name and replace it with a long-winded, technical-sounding alternative that no one has heard of.

Correct allocation of credit is important, and authoritative accounts of the history of science are useful and enlightening, but both must be balanced with science's need for consistent conventions, brevity, and public communication and outreach, especially when taxpayer's expenditures, $\rightarrow$ NATURE.COM To comment online, click on Editorials at: go.nature.com/xhunqv such as the US\$6.5 billion for the Large Hadron Collider, are at stake. Renaming the Higgs boson in the year when it is most likely to be found gets the balance wrong. (And anyway, Higgs is a better name than the God particle, isn't it?) 